\title{
The North Cumbria Community Genetics Project
}

\author{
Diana S Chase, E Janet Tawn, Louise Parker, Patricia Jonas, Claire O Parker, John Burn
}

\begin{abstract}
The aim of the North Cumbria Community Genetics Project is to establish a store of DNA, plasma, and viable cells from a cohort of around 8000 Cumbrian infants. To meet this objective, specimens of umbilical cord blood and tissue will be collected with maternal consent from babies born at the West Cumberland Hospital, Whitehaven over a five year period from January 1996. These samples will be used in a series of genetic and epidemiological studies investigating the interaction between genes, the environment, and health. There is little population movement in West Cumbria and so it will be possible to follow many of the babies throughout their childhood and to investigate the relationship between their genetic constitution and health outcome.

(F Med Genet 1998;35:413-416)
\end{abstract}

Keywords: population; gene/environment interaction; genetic and epidemiological research; DNA resource

An international effort is under way to sequence the whole of the human genome (Human Genome Project) and there are confi-

Genetics Unit, Westlakes Research Institute, Moor Row, Cumbria CA24 3JZ, UK

D S Chase

E J Tawn

C O Parker

Sir James Spence

Institute of Child

Health, University of

Newcastle, Newcastle upon Tyne NE1 4LP, UK

L Parker

Department of Human Genetics, University of Newcastle, Newcastle upon Tyne NE2 4AA, UK

$P$ Jonas

J Burn

Maternity Department, West Cumberland Hospital, Whitehaven, Cumbria CA28 3JG, UK

$\mathrm{P}$ Jonas

Correspondence to:

Dr Chase.

Received 13 October 1997 Revised version accepted for publication

2 December 1997 dent predictions that by the year 2005 the entire genome will be mapped and all the genes (about 80000 in total) will be identified. ${ }^{1}$ The next task will be to assign functions to the genes. Mutations in some genes will be sufficient in themselves to cause disease; however, many diseases, including most cancers, are the result of interactions between susceptibility genes and factors in the environment. These multifactorial diseases affect a large proportion of the population. Heart disease, for example, affects about half of the population over 65 years of age and is the single most common cause of death in this age group. ${ }^{2}$ Asthma is an increasing cause of morbidity in childhood and in 1986 affected $6.5 \%$ of children by the age of 16 years, an increase of $70 \%$ since $1974 .^{3}$ In contrast, cystic fibrosis, which is the most common autosomal recessive disorder in European populations, affects about 1 in 2500 people. ${ }^{4}$ Identification of susceptibility genes may enable population screening programmes to be considered, thus allowing subjects potentially at risk to be given appropriate lifestyle advice and regular monitoring, with the objective of reducing morbidity from disease, enhancing quality of life, and reducing premature mortality. This preventative approach has implications for a more effective use of limited health care resources. The identification of gene-disease associations and the impact of environmental factors on these associations will not be established through classical genetic family studies, but requires an epidemiological approach to the study of large, preferably unselected, population cohorts. This necessitates a source of DNA and cells from a normal population and also the ability to link results of DNA analysis with important lifestyle factors and disease outcome over an extended period of time.

To date there has been only one attempt to do this, in a study where the emphasis was primarily on child development, and although placental samples were collected DNA was not initially extracted and no viable cells were obtained. ${ }^{5}$ The North Cumbria Community Genetics Project aims to store samples of DNA, plasma, and viable cells collected from babies born at the West Cumberland Hospital, Whitehaven and to use them as a resource for future genetic epidemiological research. The West Cumbrian population is ideal for this type of research resource because not only is it relatively stable, but the study builds on the expertise in child health epidemiology and genetics already established in the area.

From the beginning it was recognised that for this innovative study, ethical debate, both with professionals and the public, would be required to gain the goodwill and support necessary for success. Before the project could proceed it required careful planning and preparation. Information leaflets, consent forms, and questionnaires were produced and the logistics of distributing these and collecting samples were established with regard to antenatal care and midwifery practices operating in the area. Technical aspects of sample collection and storage were devised and procedures developed to ensure that confidentiality of the data was safeguarded at all times. In the event it was not until January 1996, several years after the idea was initially conceived, that sample collection began.

\section{Public and professional consultation and} ethical approval

The North Cumbria Community Genetics Project was first conceived in autumn 1993 at meetings between the University of Newcastle (Department of Human Genetics and the Sir James Spence Institute of Child Health) and Westlakes Research Institute (Genetics Unit and the Department of Occupational Health and Medical Statistics). It was clear from the start that it required the full backing of the local health professionals and the support of the local community. Initial discussions with paediatric and obstetric staff at the West Cumberland Hospital concentrated on the practicalities of fitting the project into the current antenatal care programme operated by the midwifery staff. 
Early in the development of this project a presentation was made to the West Cumbria Local Research Ethics Committee to ensure that there were no fundamental ethical objections. The scientific justification was accepted by the committee who encouraged the development of a full, detailed protocol. Three months later such a protocol was presented to the committee. This encompassed methodology for the collection and storage of samples from newborns at the West Cumberland Hospital subject to informed maternal consent, together with a short lifestyle questionnaire to be completed by the mother. The project was given approval subject to a consultation period coordinated by North Cumbria Health Authority. This took the form of three meetings. The first for health professionals was chaired by a general practitioner and was addressed by members of the project team. The second two were public meetings chaired by the Chief Executive of the North Cumbria Health Authority. These were poorly attended despite associated media coverage including a brief television broadcast, a radio "phone in", as well as newspaper coverage both nationally and locally. The project was also discussed by the North Cumbria Health Authority, the West Cumbria Health Care Trust Board, and the West Cumbria Community Health Council. Feedback from the consultation period resulted in a revised protocol which was given full approval by the North Cumbria Health Authority in December 1994. A project liaison midwife was appointed in April 1995 to interface between the midwives and the research team and to develop the infrastructure for recruitment of mothers.

In all the consultations, concern centred primarily around aspects of confidentiality and a public concern about the dangers of eugenics. The rapid advances in genetic knowledge bring with them a potential danger that genetic information could be used to disadvantage people in such areas as life assurance. ${ }^{6}$ To allay such fears, full information was provided about the purpose of the study and the safeguards incorporated to ensure appropriate use of the samples and data confidentiality. Assurances were given that the public will be informed of progress on a regular basis. To this end, members of the project team regularly give presentations to local schools, women's groups, clergy, medical societies, etc. Local health professionals, in particular midwives and general practitioners responsible for antenatal care, are invited to an annual Study Day which provides an update on the project. In addition, a newsletter is distributed through public libraries, health centres, antenatal clinics, and general practitioners, and is mailed to individual people on request.

Criticism of the project has been associated almost exclusively with concern over a local issue, the involvement of the nuclear industry. It is acknowledged that without the support of British Nuclear Fuels plc (BNFL), which operates a nearby nuclear installation at Sellafield, this project could not have been initiated. BNFL is committed to the investigation of any impact its activities could potentially have on the local environment. Following the report by Gardner et al showing a statistical association between paternal preconceptional irradiation received occupationally at the Sellafield reprocessing plant and the development of childhood leukaemia, considerable effort was expended in developing child health studies in the area. Some of these studies have been reported $^{89}$ and are consistent with the realisation that the association does not represent a plausible causal mechanism. ${ }^{10}$ Factors related to the nuclear industry will of necessity be part of the environmental and occupational characteristics of the local population, but the project has a much wider remit and will focus on geneenvironment interaction in the aetiology and pathogenesis of disease. BNFL have no role in the management of the project and have not sought such a role.

Because of the unique nature of the project and the lack of published guidelines, a number of recognised UK experts with experience in the ethics of genetic and epidemiological studies were invited to form an Ethics Advisory Group. This group meets regularly and has provided valuable insight. With this input we have established a project which matches the recommendations outlined in the Statement on the Principled Conduct of Genetic Research, devised by HUGO Ethical, Legal, and Social Issues Committee and adopted by the HUGO Council in March 1996. ${ }^{11}$

\section{Confidentiality}

From the outset, confidentiality was identified as being of major importance. The project is a collaboration between the University of Newcastle and Westlakes Research Institute and this allows the separate storage of personal information and biological specimens. All the personal information is sent to Newcastle where it is stored encrypted on a stand alone computer with restricted access, while all the biological specimens are stored at Westlakes Research Institute.

Each pregnancy is allocated a different project number which allows the mother's information to be linked with her child's sample. A mother with more than one child in the project would be allocated a separate number for each pregnancy. Specimen tubes received in the laboratory do not carry any information about the baby but are identified by the project number only. This ensures that the samples are non-attributable at the point of storage and analysis.

\section{Study outline}

PROJECT DOCUMENTATION

The research team has designed three documents.

\section{Information/consent form}

This is a four page A5 leaflet which is given out to all pregnant women at the first appointment with their midwife. It gives basic information about the project in a question and answer format. There are two copies of the consent form, 
Table 1 Statement on acceptable research uses of collected samples

The primary aim of the project is to gain health benefits from the study of genes in the population. The overriding concern of the North Cumbria Community Genetics Project team is to safeguard confidentiality and minimise the possibility of harm to individual people or the community.

Every application for research using these samples will be considered with regard to current views and medical developments, and approval sought from the West Cumbria Local Research Ethics Committee.

There are three categories of study that need to be distinguished.

(1) Studies that use anonymous samples to determine the frequencies of alleles at specific loci or of polymorphism variants. Subject to (3) below, there are likely to be few ethical difficulties with the use of NCCGP samples for such work, and such anonymous samples (or DNA extracted from them) may be released to other groups subject to appropriate conditions, including the precise specification of the uses to which the samples will be put and the acknowledgement of the source of the samples. Previous discussion of such studies by the Ethics Advisory Group is not necessary, although the Group will be informed of any such arrangements that are made.

(2) Studies that involve linkage to data on individual subjects. These specific studies will be considered by the Ethics Advisory Group. Identification of individual people during the process and reporting of any such study will be done in such a way as to safeguard confidentiality.

(3) Studies that involve personality disorders, psychiatric disease, mild intellectual difficulties, and other sensitive areas. Such studies are particularly sensitive, and strong evidence of a specific health benefit would need to be shown.

one forming an integral part of the leaflet for the mother to keep and the other copy on a separate perforated page that can be returned to the midwife.

\section{Further information leaflet}

This is an eight page A5 leaflet available on request. It gives background information and the scientific rationale of the project and is aimed at midwives, general practitioners, and health visitors.

\section{Mother's questionnaire}

This is an eight page A5 booklet with questions about the health and lifestyle of the mother and her partner. An expectant mother can either complete the questionnaire at an antenatal clinic with the help of her midwife or the project liaison midwife, or she can take it home to complete.

In addition, information about the labour and birth details are abstracted from a hospital neonate form. We have designed a "Midwives' Resource Pack" which explains the practical aspects of the project. A copy of this is kept in places such as the labour ward at the West Cumberland Hospital and in health centres. It is also available to any midwife who would like a personal copy.

Copies of all documentation are available from the authors on request.

\section{INFORMED CONSENT AND ENROLMENT}

Expectant mothers receive a copy of the information/consent form from their midwife, but many women need to be reminded of the project, or to talk it over with someone before deciding whether or not to take part. The project liaison midwife provides them with a point of contact and an opportunity to discuss any queries they may have.

Consent is given for the collection and storage of samples and personal information for use in any genetic and epidemiological research study approved by the West Cumbria Local Research Ethics Committee. It was felt that although this might deter some people from taking part, it would avoid the need to contact each mother and seek permission to use her child's sample in every separate study, which would incur substantial costs and would be difficult to administer. Individual people can opt into the project as a whole but cannot opt out of specific studies. If a mother wishes to withdraw from the project she can do so at any time. The mother's consent is on behalf of the child and the consent form clearly states that research using the samples collected may involve reference to the health records of herself and her baby.

This is not a screening programme and individual results are not reported back to the participants. Diagnosis of any health related problem will still be through family doctors and the National Health Service, and is likely to be in advance of any population study using these samples. In rare and extreme circumstances when there may be an immediate major health benefit, the West Cumbria Local Research Ethics Committee would be asked to consider whether it is in the family's interest for an individual result to be disclosed.

SAMPLE COLLECTION AND PROCESSING

After the birth of the baby, the hospital midwives take specimens of cord blood and cord tissue and place them in numbered tubes which are sent to the laboratory at Westlakes Research Institute. In order to accommodate future studies, a wide variety of samples is being banked (whole blood, plasma, extracted DNA, cultured lymphocytes, and chopped cord as a source of viable cells), all of which are stored frozen.

\section{Progress}

Recruitment of pregnant women into the project began in January 1996. Currently, about $90 \%$ of pregnant women approached agree to participate. Of those giving consent, about $60 \%$ complete all or part of a mother's questionnaire. A preliminary analysis of the postcode data indicates that the distribution pattern of these women reflects the main centres of population and does not appear to vary significantly with geographical location. By November 1997, over 2500 women had been recruited, almost 2000 samples collected, and 1200 questionnaires collated.

The difficulties we have encountered so far have largely been of a practical nature. There is no central booking system in West Cumbria and pregnant women can attend one of many antenatal clinics at various geographical locations. The current trend is towards community based antenatal care: many pregnant women now carry their own obstetric notes and may only come to a hospital clinic once or twice in their first pregnancy, and possibly not at all for subsequent uncomplicated pregnancies. The project liaison midwife has proved to be essential in providing the interface for recruitment and overcoming these difficulties. Occasionally samples have not been collected by the midwives owing to the uneven work load on the labour ward, unexpected complications, or an emergency delivery, but this is rare. 


\section{Future studies}

A wide range of health related research studies could benefit from access to these samples. In consultation with the Ethics Advisory Group, a "Statement on Acceptable Research Uses of Collected Samples" has been drawn up (table 1). Some studies may use the samples totally anonymously, for example to estimate the number of people in the population who have a specific genetic polymorphism. Other studies may need to link laboratory test data with information contained in the mother's questionnaire or with medical records of the mother or child. Whatever type of study is undertaken, the results will be published such that no individual person can be identified.

Currently, two research studies have been approved by the West Cumbria Local Research Ethics Committee. One uses the glycophorin-A assay and estimates the number of somatic mutations affecting the gene for the $M N$ blood group antigen. The other is a study of a microdeletion on chromosome 22, associated with DiGeorge syndrome. The results of each study will be reported at a later date.

\section{Conclusion}

The increasing awareness of the importance of gene-environment interaction in the aetiology of a wide range of diseases has led to a need for population based studies capable of linking epidemiological, genetic, and health outcome data. The North Cumbria Community Genetics Project has been set up to meet this objective.
The authors acknowledge the help of the Ethics Advisory Group whose members currently are Dr Angus Clarke, Dr Martin whose members currently are Dr Angus Clarke, Dr Martin Professor Roger Berry, particularly in the initiation of the project, is greatly appreciated. We wish to thank Dr Paul Danie Mr Julian Smith, Mrs Gail Barker, and Mrs Joan Killip for thei support in the development and execution of the project. We are indebted to the community and hospital based midwifery team without whom this project would not be possible, to $\mathrm{Mr}$ Stephe Bober and Dr Sheila Precious from the West Cumberland Hospital for their support, and to our many colleagues and member of the public who have contributed in helping to ensure a thorough exploration of the ethical and practical issues addressed in this project. This project is supported by British Nuclear Fuel plc, Westlakes Research Institute, and the North of England Children's Cancer Research Fund.

1 Jones SL. Genetics: changing health care in the 21 st century. f Obstet Gynecol Neonatal Nurs 1996;25:777-83.

2 Central Statistics Office. Social trends. London: HMSO 1996.

3 Lewis S, Butland B, Strachan D, et al. Study of the aetiology of wheezing illness at age 16 in two national British birth cohorts. Thorax 1996;51:670-6.

4 Traystman MD, Schulte NA, MacDonald M, Anderson JR, Sanger WG. Mutation analysis for cystic fibrosis to determine the carrier status in 167 sperm donors from the Nebraska Genetic Semen Bank. Hum Mutat 1994;4:271-5.

5 Farrow A, Farrow SC, Little R, Golding J. The repeatability of self-reported exposure after miscarriage. ALSPAC Study Team. Avon Longitudinal Study of Pregnancy and Childhood. Int f Epidemiol 1996;25:797-806.

6 Nuffield Council on Bioethics. Genetic screening - ethical issues. London: Nuffield Council on Bioethics, 1993.

7 Gardner MJ, Snee MP, Hall AJ, Powell CA, Downes S, Terrell JD. Results of case-control study of leukaemia and rell JD. Results of case-control study of leukaemia and lymphoma among young people near Sellafield nucle

power plant in West Cumbria. BMF 1990;300:423-9.
8 Parker L, Craft AW, Smith J, et al. Geographical distribution of preconceptional radiation doses to fathers employed a the Sellafield nuclear installation, West Cumbria. BMJ 1993;307:966-71.

9 Wakeford R, Parker L. Leukaemia and non-Hodgkin's lymphoma in young persons resident in small areas of West Cumbria in relation to paternal preconceptional irradiation. Br $\mathcal{F}$ Cancer 1996; 73:673-9.

10 Doll R, Evans HJ, Darby SC. Paternal exposure not to blame. Nature 1994;367:678-80.

11 Knoppers BM, Hirtle M, Lormeau S. Ethical issues in international collaborative research on the human genome: the HGP and the HGDP. Genomics 1996;34:272-82. 